\title{
Resonant band-electron $-f$-electron scattering theory for highly correlated actinide systems
}

\author{
Gong-Jia Hu, Nicholas Kioussis, ${ }^{*}$ Amitava Banerjea, ${ }^{\dagger}$ and Bernard R. Cooper \\ Department of Physics, West Virginia University, Morgantown, West Virginia 26506
}

(Received 22 January 1988)

\begin{abstract}
In earlier studies we recognized that the highly correlated behavior of the $f$ electrons within moderately delocalized light actinide (uranium, neptunium, plutonium) systems is linked to the non- $f$-band behavior via the hybridization process. By transforming the hybridization into a bandelectron $-f$-electron resonant scattering from the correlated multiplet states of the actinide ions, and considering only the scattering processes that involve $f$ electrons in the $m_{l}=0, m_{s}= \pm \frac{1}{2}$ states (for quantization along the interionic axis) which dominate the two-ion interactions, our earlier work explained the main features of the anisotropic magnetic equilibrium behavior for the PuSb system but failed to reproduce the correct polarization (longitudinal) for the long-period antiferromagnetic structure observed in the temperature range below the Néel temperature. In this paper we include the next-to-dominant scattering channels (single-site scattering processes involving $f$ electrons with $\left.m_{l}= \pm 1, m_{s}=\mp \frac{1}{2}\right)$. This refinement changes the angular dependence of the anisotropic interaction, and successfully yields the ferromagnetic to longitudinally polarized long-period antiferromagnetic phase transition as is experimentally observed. Excellent agreement with experiment for the correlation length anisotropy is also obtained. For the magnetic excitation behavior in the ferromagnetic phase pertinent to $\mathrm{PuSb}$ at $T=0$, the theory gives a spectrum with two polarized branches at the zone boundary for $\mathbf{q}$ along the [100] (transverse-to-moment) direction. In fact, the predicted excitation behavior is rather remarkable. The appearance of two polarized branches rather than a single branch at the zone boundary occurs only over an extremely narrow range of crystal-field splitting. We choose the crystal-field splitting to give two branches, and this unadjustably yields excitation energies that are very close to the experimental values. An only slightly different crystal-field value would give neither two branches nor correct excitation energies.
\end{abstract}

\section{INTRODUCTION}

Recently, basic understanding of the highly correlated magnetic behavior of the cerium monopnictide systems has been achieved. ${ }^{1-4}$ The theory is based on an anisotropic two-ion interaction that arises from the hybridization of band electrons with moderately delocalized $f$ electrons. This hybridization was first treated by Coqblin and Schrieffer ${ }^{5}$ to explain the behavior of dilute cerium alloys by transforming it into a band- $f$ resonant scattering from the multiplet states of the $\mathrm{Ce}^{3+} f^{1}$ ions, and was subsequently extended by Cooper and Siemann ${ }^{1}$ to treat the hybridization-mediated anisotropic two-ion coupling. For $\mathrm{Ce}^{3+} f^{1}$ lattices, it has been shown that the predominant two-ion coupling comes from the $m_{l}=0$ (with respect to the interionic axis) part of each $f^{1}$ localized wave function., ${ }^{3,4,6}$ Extending this theory to the general case of $f$ ions with more than one $f$ electron in each ion, Cooper et al. ${ }^{6-10}$ found that the angular dependence of the hybridization-mediated anisotropic two-ion interaction depends on the $f$ occupation number and on the nature of the intraionic coupling. They used PuSb (which has $\mathrm{NaCl}$ structure) as a model system to test the theory and reported the results of calculations for a $\mathrm{Pu}^{3+} f^{5} \mathrm{fcc}$ lattice system in the cases of $j-j$ coupling, $L-S$ coupling, and intermediate coupling (IC). ${ }^{9}$ Success was achieved in explaining the main features of the anisotropic magnetic equilibrium behavior, namely, the behavior of the magne- tization with temperature and the phase transition from a ferromagnetic phase to a long-period antiferromagnetic phase; but the theory ${ }^{9,10}$ failed to reproduce the correct polarization (longitudinal with respect to the modulation direction) for the long-period antiferromagnetic structure observed $^{11,12}$ in the temperature range below the Néel temperature. The theory also gave a susceptibility singularity ${ }^{13}$ in the near-critical region corresponding to the incorrect polarization. For the excitation behavior, the theory failed to explain why for PuSb there are ${ }^{14}$ two modes with the wave vector perpendicular to the magnetic moment (these modes are at least partially polarized, parallel, and perpendicular to the wave vector, respectively).

In the original calculations ${ }^{1,2,6}$ for the $\mathrm{Ce}^{3+}$ ion, which has only one $f$ electron, it was shown ${ }^{6}$ that to the lowest order in $1 / k_{F} R$ the dominant contribution to the two-ion interaction comes from processes in which the band electron involved in the associated single-site scattering event had magnetic quantum number $m= \pm \frac{1}{2}$ (using the interionic axis as the axis of quantization). [Here $k_{F}$ is the Fermi wave vector for a free-electron-like gas, $R$ is the separation between ions, and $1 / k_{F} R$ is the characteristic expansion parameter in Ruderman-Kittel-Kasuya-Yosida (RKKY)-type theories, i.e., theories where interactions between widely separated ions are mediated by scattering with an intervening electron gas.] Furthermore, it was recognized $^{2,6}$ that the $m_{l}=0$ component of the scattered 
electron provided the physical basis for this dominance, because scattering events in which the scattered electron has $m_{l}=0$ require that the electron within the localized ion has $m_{l}=0$. This causes the ionic charge to be "sucked out" along the interionic axis, and gives rise to a situation where the two ions interact via cooperative hybridization with the intervening band sea. This then provided a physical rationale for adopting ${ }^{6,9}$ an $m_{l}=0$ scattering event "selection rule" for scattering events providing a coupling between $f^{n}$ (light actinide) ions. Namely, if we express free-ion states by determinantal products (Slater determinants) of single-electron $(l=3)$ wave functions labeled by quantum number $m_{l}$ and $m_{s}$, it was argued $^{6,9}$ that the dominant contribution to the twoion interaction arose from scattering processes involving $f$ electrons with $m_{l}=0$, as these were the states that "pointed" their charge densities along the interionic axis (which was taken as the axis of quantization). However, $m= \pm \frac{1}{2}$ states not only have contributions from $m_{l}=0$, $m_{s}= \pm \frac{1}{2}$ states, but also have contributions from states which have $m_{l}= \pm 1, m_{s}=\mp \frac{1}{2}$. The latter are states that point their charge densities off the interionic axis. Actually, both the $m_{l}=0$ and $m_{l}= \pm 1$ contributions to the $m= \pm \frac{1}{2}$ states were included for the $\mathrm{Ce}^{3+}$ theory. However, in the initial work extending the theory to systems with more than one $f$ electron per ion, Cooper et al. ${ }^{6,8}$ included only the $m_{l}=0$ component. In considering the source of the discrepancies between theory and experiment for $\mathrm{PuSb}$, we recognized ${ }^{15}$ that the assumption of including only $m_{l}=0$ contributions is not complete; and the $m= \pm \frac{1}{2}$ states with $m_{l}= \pm 1$, partially included for the $\mathrm{Ce}^{3+}$ theory, may play a crucial "fine tuning" role in the anisotropy of the two-ion interaction between $f^{n}$ (light actinide) ions.

In this paper we will describe calculations including the next-to-dominant single-site scattering processes, involving $f$ electrons in $m_{l}= \pm 1, m_{s}=\mp \frac{1}{2}$ states (i.e., those $m_{l}= \pm 1$ states which contribute to $m= \pm \frac{1}{2}$ in the $j-j$ coupling limit). PuSb is used as a model system to test the theory. In Sec. II we briefly review the model of hybridization-mediated interionic coupling in the $f^{n}$ system and the theory for the magnetic excitations. In Sec. III we illustrate the calculation of the scattering coefficients in the case of both $L-S$ coupling and intermediate coupling (IC) on including the additional $m_{l}= \pm 1$ scattering processes. Results and discussion are presented in Sec. IV.

\section{MODEL AND FORMALISM}

The Anderson model ${ }^{16}$ is used ${ }^{5,17}$ in describing the hybridization between the quasilocalized $f$ and the band electrons. The mixing Hamiltonian can be written as

$$
H_{\operatorname{mix}}=\sum_{k, m}\left(V_{k} b_{k m}^{\dagger} c_{m}+V_{k}^{*} c_{m}^{\dagger} b_{k m}\right) \text {. }
$$

Here, $V_{k}$ is the strength of the mixing potential, and $b^{\dagger}$ (b) and $c^{\dagger}(c)$ denote creation (destruction) operators for the band and ionic states, respectively. The mixing potential is assumed to be spherical, and resonant mixing occurs between the localized $f$ electrons and the $f$ partial-wave components of the bands. Upon applying the Schrieffer-Wolff ${ }^{18,19}$ transformation, the hybridization (mixing) interaction transforms to an effective electron scattering Hamiltonian, which was first developed by Coqblin and Schrieffer ${ }^{5}$ for cerium impurity ions. When this scattering Hamiltonian is treated to second order as a perturbation, it yields the hybridization-mediated anisotropic two-ion interaction, which is generically related to the Ruderman-Kittel-Kasuya-Yosida (Ref. 20) interaction, but acts via the orbital ${ }^{21,22}$ rather than the spin moment. The two-ion interaction theory for $\mathrm{Ce}^{3+}$ lattices was developed by Cooper et al. ${ }^{1,2,6}$ for cerium compounds.

To treat the single-site band- $f$ hybridization in the $f^{n}$ case, Thayamballi and Cooper ${ }^{7}$ extended the singleelectron exchange scattering methodology developed by Coqblin and Schrieffer ${ }^{5}$ for the $f^{1}$ case. They wrote the single-site scattering Hamiltonian as

$$
\mathscr{H}_{n s}=-\sum_{k, k^{\prime}} g_{k k^{\prime}} \sum_{M, M^{\prime}} \sum_{m, m^{\prime}} A_{m m^{\prime}}^{M M^{\prime}} d_{M^{\prime}}^{\dagger} d_{M} b_{k^{\prime} m^{\prime}}^{\dagger} b_{k m} \text {, }
$$

where $A_{m m^{\prime}}^{M M^{\prime}}$ are the scattering coefficients, ${ }^{6,7} M(m)$ and $M^{\prime}\left(m^{\prime}\right)$ are the initial- and final-state magnetic quantum numbers of the ion (band electron), $d^{\dagger}(d)$ is the creation (destruction) operator for the appropriate ionic state, and $g_{k k^{\prime}}$ is the coupling coefficient which depends on the mixing strength $\left|V_{k}\right|^{2}$ and the energies of the initial, final, and intermediate states. Following Coqblin and Schrieffer, ${ }^{5}$ we assume that $g_{k k^{\prime}}$ is a constant for $|\mathbf{k}|$, $\left|\mathbf{k}^{\prime}\right| \simeq k_{F}$, and is zero otherwise. If we define new scattering coefficients which retain only the exchange and relative direct scattering contributions,

$$
\mathcal{A}_{m m^{\prime}}^{M M^{\prime}}=A_{m m^{\prime}}^{M M^{\prime}}-\frac{\delta_{M M^{\prime}} \delta_{m m^{\prime}}}{(2 J+1)} \sum_{N} A_{m m}^{N N},
$$

then treating the single-site scattering Hamiltonian to second order one gives the interaction between ions at sites $i$ and $j$ as

$$
\mathscr{H}_{i j}=-E_{i j} \sum_{M, M^{\prime}} \sum_{N N^{\prime}} J_{M M^{\prime}}^{N N^{\prime}} L_{M^{\prime} M}^{(i)} L_{N^{\prime} N}^{(j)}
$$

where

$$
J_{M M^{\prime}}^{N N^{\prime}}=\sum_{m, m^{\prime}} \mathcal{A}_{m m^{\prime}}^{M M^{\prime}} \mathcal{A}_{m^{\prime} m}^{N N^{\prime}}
$$

and

$$
L_{\alpha \beta}=|\alpha\rangle\langle\beta| \text {. }
$$

The indices $M, M^{\prime}, N, N^{\prime}$ are summed over all possible $M$ values for the ground-state multiplet of the ion, and $m, m^{\prime}$ are summed over all possible $m$ values. $E_{i j}$ is a range function for the two-ion anisotropic exchange, which for free-electron bands is identical with the RKKY range function. ${ }^{6}$ Here we treat $E_{i j}$ as phenomenological exchange parameters with $E_{n}$ giving the strength of the interaction with the $n$th nearest neighbors.

Note that the ionic states are labeled by their magnetic quantum numbers with quantization along the interionic axis. In order to treat magnetic ordering in a lattice of ions, all such states must be transformed to a common 
quantization axis through the appropriate transformation. ${ }^{1,23}$ Then the two-ion interaction Hamiltonian of the system can be written as ${ }^{6}$

$$
\mathscr{H}=-\sum_{\mathbf{R}_{i j}} \sum_{\mu \nu \epsilon \sigma} J_{\mu \nu}^{\epsilon \sigma}\left(\mathbf{R}_{i j}\right) L_{\mu \nu}^{(i)} L_{\epsilon \sigma}^{(j)},
$$

where $\mu, v, \epsilon$, and $\sigma$ refer to the $M$ states, and

$$
J_{\mu \nu}^{\epsilon \sigma}\left(\mathbf{R}_{i j}\right)=E_{i j} B_{\mu \nu}^{\epsilon \sigma}(\theta) e^{-i(\mu-v+\epsilon-\sigma) \phi_{i j}}
$$

with

$$
B_{\mu \nu}^{\epsilon \sigma}(\theta)=\sum_{M M^{\prime}} \sum_{N N^{\prime}} J_{M M^{\prime}}^{N N^{\prime}} d_{\mu M^{\prime}}(\theta) d_{v M}(\theta) d_{\epsilon N}(\theta) d_{\sigma N^{\prime}}(\theta)
$$

Here $\theta$ and $\phi$ are the polar and azimuthal angles of the interionic axis $\mathbf{R}_{i j}$ with respect to the axis of quantization chosen along a cube-edge direction in the crystal, and $d_{\alpha \beta}(\theta)$ is the rotational transformational matrix as is conventionally defined [see Ref. 23, Eq. (5.35)]. In order to treat the equilibrium magnetic ordering behavior, this two-ion interaction [Eq. (7)] is treated in the mean-field approximation. The mean-field (MF) Hamiltonian is given by

$$
\mathcal{H}_{\mathrm{MF}}=\sum_{i} \sum_{n} \epsilon_{n}^{i} L_{n n}^{(i)}
$$

where $\epsilon_{n}^{i}$ denotes the energy of the MF state $|n\rangle$ on site $i$.

To study the magnetic excitations of the system we project the Fourier transform of the Hamiltonian defined in Eq. (7) into the MF manifold. At $T=0$ we need to consider the set of operators that take the system from, or to, the MF ground state $\left(L_{1 n}\right.$ or $\left.L_{n 1}, n \neq 1\right)$. Using the random-phase approximation (RPA) and the commutation relation ${ }^{2}$

$$
\left(\mathcal{H}_{M F}, L_{n n^{\prime}}^{\mathrm{q}}\right)=\left(\varepsilon_{n^{\prime}}-\varepsilon_{n}\right) L_{n n^{\prime}}^{\mathrm{q}},
$$

we get the equation of motion

$$
\left[\mathcal{H}-\mathcal{H}_{\mathrm{MF}}, L_{n n^{\prime}}^{\mathrm{q}}\right]=2\left(\left\langle L_{n n}^{0}\right\rangle-\left\langle L_{n^{\prime} n^{\prime}}^{0}\right\rangle\right) \sum_{s, t} J_{n^{\prime} n}^{s t} L_{s t}^{\mathrm{q}}
$$

The thermal average $\left\langle L_{n n}^{0}\right\rangle$ is unity if $n$ is the ground state $(n=1)$ and zero otherwise. On diagonalizing the resulting $10 \times 10$ dynamical matrix [upward and downward transitions between the ground state and each of the five excited states for a $J=\frac{5}{2}$ system, such as $\left.\mathrm{Pu}^{3+}\left(f^{5}\right)\right]$, we get five modes at energy gain and five at energy loss.

\section{CALCULATION OF SCATTERING COEFFICIENTS}

In this section we review the calculation of the scattering coefficients $A_{m m^{\prime}}^{M M^{\prime}}$ for the $f^{n}$ systems. In the $f^{1}$ case it has been shown ${ }^{1,6}$ that the predominant two-ion coupling, which arises from the hybridization between the ion and the band electrons, which is treated by considering resonant scattering of a band electron by the ion, comes from the $m= \pm \frac{1}{2}$ (with respect to interionic axis) part of each $f$ localized wave function. To extend this theory to $f^{n}$ systems, our basic assumption ${ }^{6,7,9}$ is that the single-site scattering event consists of a one electron (out of the many-electron ionic state) exchange only; all the quantum numbers of all the other electrons remain the same; and the scattering being a single-electron process does not affect the other electrons. In addition, in the previous work ${ }^{9,10}$ for $\mathrm{Pu}^{3+} f^{5}$ systems we have proceeded on the basis that only those events in which the scattering electron has $m_{l}=0\left(m= \pm \frac{1}{2}\right)$ contribute to maximum interionic coupling; and therefore we have included only these dominant scattering events in finding the two-ion interaction. In order to have the background necessary to follow what is involved in including the $m_{l}= \pm 1$ nextto-dominant scattering channels, we now summarize the way in which the scattering coefficients have been calculated. The scattering coefficient $A_{m m^{\prime}}^{M M^{\prime}}$ corresponds to first adding (removing) a band (localized) electron with magnetic quantum number $m$ to (from) the state $\left|J, M^{\prime}\right\rangle$ to produce an $f^{n+1}\left(f^{n-1}\right)$ intermediate state, and then removing (adding) a localized (band) electron with magnetic quantum number $m^{\prime}$ to give the final state $\left|J, M^{\prime}\right\rangle$, and is given by, 6,7

$$
A_{m m^{\prime}}^{M M^{\prime}}=\left(|V|^{2}\right)^{-1}\left\langle f^{n}\left(M^{\prime}, m\right) B_{k^{\prime}}\left(m^{\prime}\right)\left|\mathcal{H}_{\text {mix }}\right| f^{n \pm 1}\left(m, m^{\prime}\right)\right\rangle\left\langle f^{n \pm 1}\left(m, m^{\prime}\right)\left|\mathcal{H}_{\text {mix }}\right| f^{n}\left(M, m^{\prime}\right) B_{k}(m)\right\rangle
$$

Here $B_{k}(m)$ is a band state with wave vector $\mathbf{k}$, and has an $f$ spherical component about the scattering site with magnetic quantum number $m$. $f^{n}(M, m)$ denotes an ion of configuration $f^{n}$ with magnetic quantum number $M$ and with one of the electrons within the ion with magnetic quantum number $m$. $f^{n \pm 1}\left(m, m^{\prime}\right)$ are intermediate states which contain electrons with magnetic quantum numbers $m$ and $m^{\prime}$. To find the matrix elements of $\mathscr{H}_{\text {mix }}$ entering Eq. (13), the $f^{n}$ and $f^{ \pm 1}$ wave functions have to be written in terms of linear combinations of Slater determinants (SD) of one-electron wave functions. To simplify the calculations, some assumptions, consistent with the physics involved, were made. First, it was assumed that the initial and final states of the $f^{n}$ ion are in its Hund's rule favored- $J$ manifold, in which the ionic states (labeled by $|J, M\rangle)$ are assumed degenerate in the absence of $\mathscr{H}_{\text {mix }}$. Second, due to the large Coulomb correlation energy that is involved in adding or removing an $f$ electron in the initial (or the final) state, we neglected the splitting within the intermediate-state configuration. This means that the formation of the intermediate state will only be governed by the considerations of the exclusion principle, and one can sum over all possible multiplets of the $f^{n+1}$ or the $f^{n-1}$ ion in the intermediate state. In other words, the intermediate states need not be explicitly written in terms of linear combinations of Slater determinants and can be treated as virtual states.

For a $j-j$ coupled $^{6,7}$ ionic state, the $f^{n}$ wave functions 
are written in terms of $n \times n$ Slater determinants of the one-electron states in the $(j, m)$ representation. The $A_{m m^{\prime}}^{M M^{\prime}}$ are then found by using Eq. (13), together with the restrictions imposed by the exclusion principle. An ex- ample of this procedure is given in Refs. 6 and 7.

In the $L-S$ coupled case, the $n$ electron $|J, M\rangle$ wave function is written in terms of $\left|L, S, M_{L}, M_{S}\right\rangle$ wave functions coupled by appropriate Wigner coefficients ${ }^{24}$

$$
|J, M\rangle=\sum_{M_{L}}(2 J+1)^{1 / 2}(-1)^{L-S+M-2 J}\left[\begin{array}{ccc}
L & S & J \\
M_{L} & M-M_{L} & M
\end{array}\right]\left|L S M_{L} M_{S}\right\rangle
$$

The scattering amplitude $A_{m m^{\prime}}^{M M^{\prime}}$ is then given by

$$
A_{m m^{\prime}}^{M M^{\prime}}=(2 J+1) \sum_{M_{L}, M_{L}^{\prime}}(-1)^{M+M^{\prime}-2 S}\left(\begin{array}{ccc}
L & S & J \\
M_{L} & M-M_{L} & M
\end{array}\right)\left(\begin{array}{ccc}
L & S & J \\
M_{L}^{\prime} & M^{\prime}-M_{L}^{\prime} & M^{\prime}
\end{array}\right) a\left(M_{L}, M_{S} ; M_{L}^{\prime}, M_{S}^{\prime}, m, m^{\prime}\right),
$$

where $a\left(M_{L}, M_{S} ; M_{L}^{\prime}, M_{S}^{\prime} ; m, m^{\prime}\right)$ is the amplitude of a scattering event in which the ionic state changes from $\left|M_{L}, M_{S}\right\rangle$ to $\left|M_{L}^{\prime}, M_{S}^{\prime}\right\rangle$ (the quantum numbers $L$ and $S$ having been suppressed for brevity) with the exchange of an electron with magnetic quantum number $m$ for one with $m^{\prime}$. In order to compute the amplitudes $a\left(M_{L}, M_{S} ; M_{L}^{\prime}, M_{S}^{\prime} ; m, m^{\prime}\right)$ the $\left|M_{L}, M_{S}\right\rangle$ states need to be further decomposed into Slater determinants (SD) of one-electron wave functions labeled by $m_{l}$ and $m_{s}$. Then the scattering amplitude $a\left(M_{L}, M_{S} ; M_{L}^{\prime}, M_{S}^{\prime} ; m, m^{\prime}\right)$ is obtained by multiplying the coefficient of the SD containing $m_{l}=0, m_{s}=\frac{1}{2}$, or $m_{s}=-\frac{1}{2}\left(m_{l}+m_{s}=m\right)$ in the wave function $\left|M_{L}, M_{S}\right\rangle$ with that of the SD containing $m_{l}^{\prime}=0, \quad m_{s}^{\prime}=\frac{1}{2}, \quad$ or $\quad m_{s}^{\prime}=-\frac{1}{2} \quad\left(m_{l}^{\prime}+m_{s}^{\prime}=m^{\prime}\right) \quad$ in $\left|M_{L}^{\prime}, M_{S}^{\prime}\right\rangle$. Note that all the quantum numbers of all the other electrons in the two SD's involved remain unchanged and are not affected by the single-electron scattering process. It is this step in the calculation that is charged to include other scattering channels such as those involving $m_{l}= \pm 1$. This then changes the scattering coefficients and hence the angular dependence of the two-ion interaction.

For the case of intermediate coupling (IC) the calculations are similar in principle to those in the $L-S$ coupling case except that the ground state is different, i.e., the ground state is a linear combination of $L-S$ terms. For the $\mathrm{Pu}^{3+} f^{5}$ system the ground state for $L-S$ coupling is the predominant component of the IC ground state, and the dominant components are ${ }^{9,10,25}$ one ${ }^{6} H(\sim 67 \%)$ and four ${ }^{4} G(\sim 25 \%)$ terms. The ground-state wave function $|J M\rangle$ with a given value of $M$ may be approximately written as ${ }^{9}$

$$
\left.\left.|J M\rangle=C_{H} \mid{ }^{6} H_{5 / 2} M\right)+C_{G} \sum_{\alpha=1}^{4} y_{\alpha} \mid \alpha^{4} G_{5 / 2} M\right),
$$

where $\alpha \equiv W U$ distinguishes the four ${ }^{4} G$ states present in the IC ground state; $W$ is used to label the irreducible representations of $G_{2}$, while $U$ labels the irreducible representation of $R_{7}$. Coefficients $y_{\alpha}$ can be calculated ${ }^{24,26}$ by diagonalizing the matrices for the Casimir operators of the $G_{2}$ and $R_{7}$ groups along with $L^{2}$, which is also the Casimir operator for the group $R_{3}$, and $S^{2}$; and coefficients $C_{H}$ and $C_{G}$ are obtained by diagonalizing the sum of the Coulomb and spin-orbit matrices in the basis of the ${ }^{6} \mathrm{H}$ and four ${ }^{4} \mathrm{G}$ states.

Cooper et al. ${ }^{6,7,9,10}$ have reported the results of calculations for the $\mathrm{Pu}^{3+} f^{5}$ system in the cases of $j-j$ coupling, $L-S$ coupling, and IC. They found that in the limit of $j-j$ coupling, the longitudinally polarized antiferromagnetic phase, which is observed experimentally ${ }^{12}$ in the temperature range below the Neel temperature, is preferred over the transversely polarized one, while in cases of both $L-S$ coupling and IC the antiferromagnetic phase is transverse polarized. Thus to understand what is necessary to obtain the experimentally observed ${ }^{12}$ longitudinal polarization, it is worthwhile to investigate the detailed scattering processes in the $j-j$ coupling limit even though the free-ion ground state of $\mathrm{Pu}^{3+}$ in $\mathrm{PuSb}$ is close to the $L-S$ coupling ${ }^{6} \mathrm{H}$ ground state. As discussed in Sec. $I$, in the $j-j$ coupling limit the dominant contribution to the two-ion interaction arises from those single-site scattering processes involving $f$ electrons in the $m= \pm \frac{1}{2}$ states. Actually, these events include three single-site scattering channels,

$$
\begin{aligned}
& \text { (1) } m_{l}=0, \quad m_{s}= \pm \frac{1}{2} \leftrightarrow m_{l}^{\prime}=0, \quad m_{s}^{\prime}= \pm \frac{1}{2} ; \\
& \text { (2) } m_{l}=0, \quad m_{s}= \pm \frac{1}{2} \leftrightarrow m_{l}^{\prime}= \pm 1, \quad m_{s}^{\prime}=\mp \frac{1}{2} ; \\
& \text { (3) } m_{l}= \pm 1, \quad m_{s}=\mp \frac{1}{2} \leftrightarrow m_{l}^{\prime}= \pm 1, \quad m_{s}^{\prime}=\mp \frac{1}{2} .
\end{aligned}
$$

To treat channels (2) and (3) in the $L-S$ and IC cases, it is necessary to take into account appropriate Clebsch-Gordan coefficients when we calculate the scattering amplitude. ${ }^{15}$

$$
\begin{aligned}
a\left(M_{L}, M_{S} ; M_{L}^{\prime}, M_{S}^{\prime} ; m_{j}, m_{j}^{\prime}\right)=\sum_{m_{l}, m^{\prime}} & 6(-1)^{m+m^{\prime}}\left[\begin{array}{ccc}
l & s & \frac{5}{2} \\
m_{l} & m-m_{l} & m
\end{array}\right]\left[\begin{array}{ccc}
l & s & \frac{5}{2} \\
m_{l}^{\prime} & m^{\prime}-m_{l}^{\prime} & m^{\prime}
\end{array}\right] \\
& \times a\left(M_{L}, M_{S} ; M_{L}^{\prime}, M_{S}^{\prime} ; m_{l}, m_{s}, m_{l}^{\prime}, m_{s}^{\prime}\right)
\end{aligned}
$$


where $l=3, s=\frac{1}{2}, m_{s}=m-m_{l}$, and $m_{s}^{\prime}=m^{\prime}-m_{l}^{\prime}$.

In orders of ${ }^{6} 1 / k_{F} R$ the leading contribution to the two-ion interaction comes from channel (1) which was the only channel considered in the initial studies ${ }^{9,10}$ for the $L-S$ and IC cases. The next two higher-order contributions, ${ }^{6}$ arising from channels (2) and (3), which were not included in earlier studies, 9,10 therefore should play a key role in yielding the longitudinal polarization of the antiferromagnetic phase of the fcc $\mathrm{Pu}^{3+}$ system as occurs in PuSb. On including these two scattering channels in our calculations, we have obtained some striking results and will describe them in the next section.

\section{RESULTS AND DISCUSSION}

PuSb shows magnetic structures with strong [001] anisotropy. ${ }^{8,11,12}$ At low temperature it is a [001] ferromagnet and undergoes a first-order phase transition to a longitudinal polarized (modulation wave vector parallel to the moment direction) long-period antiferromagnet at ${ }^{12}$ about $67 \mathrm{~K}$; it becomes paramagnetic at a Néel temperature $T_{N}=85 \mathrm{~K}$. At $10 \mathrm{~K}$ in the ferromagnetic phase, an almost dispersionless magnetic transition with an energy of $4.3 \mathrm{THz}(206 \mathrm{~K})$ was observed by neutron inelastic scattering studies. ${ }^{14}$ At the zone boundary for the wave vector $\mathbf{q}=[1,0,0]$ (transverse to the magnetization) there exist two excitations: the mode at $4.3 \mathrm{THz}(206 \mathrm{~K})$ polarizes along the [010] direction (transverse to both the magnetization and the wave vector), whereas the mode at 3.5 THz (168 K) has polarization along the wave vector ([100] direction). At $[0.7,0,0]$ two separate modes can no longer be distinguished. Including the two next-todominant scattering channels [channels (2) and (3) of Eq. (17)] in calculating the scattering amplitude allows us to understand these static and dynamic magnetic properties, except for the dispersionlessness character of the excitation spectrum across much of the Brillouin zone, and also allowed us to predict ${ }^{21}$ the anisotropy in correlation lengths as recently observed. ${ }^{27}$

\section{A. Equilibrium magnetic behavior}

Figure 1 shows the polarization diagram in the $W_{1}$ versus $W_{2}$ plane; i.e., this shows whether the long-period antiferromagnetic phase has transverse or longitudinal polarization, i.e., alignment of ordered moments with respect to modulation direction. (For modeling purposes, the long-period antiferromagnetic phase was taken to have ferromagnetic [001] planes in a three-up-threedown $(+++-\ldots)$ arrangement.) Here the phenomenologically introduced weighting factor $W_{1}\left(W_{2}\right)$ measures the ratio of the magnitude of contribution to the scattering amplitude from channel 2 (3) compared to that from channel 1 .

The behavior shown includes all possibilities going from the case of vanishing contributions from channels 2 and $3\left(W_{1}=W_{2}=0\right)$ up to the case where each of those channels has a contribution to the scattering coefficients that is equal to that of channel $1\left(W_{1}=W_{2}=1\right)$. The calculation has been performed for $L-S$ coupled $\mathrm{Pu}^{3+}$. For each value of $W_{1}$ and $W_{2}$, the ratio of next-nearest to nearest-neighbor anisotropic exchange parameters,

$$
\begin{aligned}
& \mathrm{Pu}^{3+}\left(f^{5}\right), L-S \text { coupling } \\
& \mathrm{W}_{1}:\left(\mathrm{m}_{l}=0 \rightarrow \mathrm{m}_{l}= \pm 1\right) \\
& \mathrm{W}_{2}:\left(\mathrm{m}_{l}= \pm 1 \longrightarrow \mathrm{m}_{l}= \pm 1\right)
\end{aligned}
$$

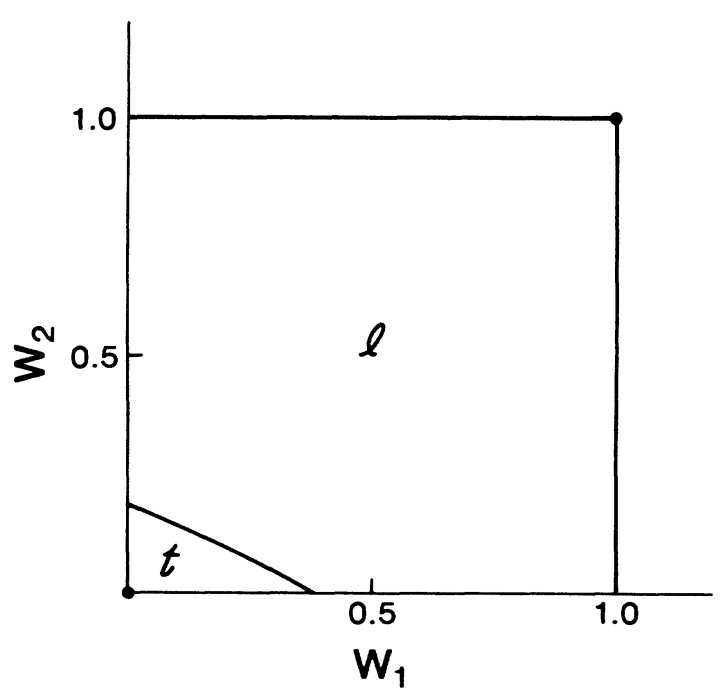

FIG. 1. Polarization behavior as a function of the scattering channel weightings $W_{1}$ and $W_{2}$ in the long-period antiferromagnetic phase for parameters characteristic of $\mathrm{PuSb}$. The longitudinal and transverse polarized phases are labeled by $l$ and $t$, respectively.

$E_{2} / E_{1}$, has been taken as unity; $E_{3} / E_{1}$ has been chosen to give the experimental ratio of the Néel temperature $(85$ $\mathrm{K})$ to the ferromagnetic transition temperature $(67 \mathrm{~K})$ for $\mathrm{PuSb} ; E_{1}$ has been chosen to give the experimental $T_{N}$; and the crystal-field parameter $60 \mathrm{~B}_{4}$ has been chosen to give the magnetic excitation intensity behavior described in Sec. IV C. The variation in $E_{3} / E_{1}$ values as $W_{1}$ and $W_{2}$ vary is small. $\left(E_{3} / E_{1}\right.$ varies between about -0.25 and -0.40 , while $E_{1}$ is always within a few percent of $150 \mathrm{~K}$.) As shown, there is a critical line which separates the $W_{1}$ versus $W_{2}$ plane into regions of longitudinal (l) and transverse $(t)$ polarization for the antiferromagnetic state. (The critical line runs between $W_{1}=0.38, W_{2}=0$ and $W_{1}=0, W_{2}=0.19$. On the critical line, the antiferromagnetic phase is unpolarized.) Except for the small region with $W_{1}$ and $W_{2}$ values less than those on the critical line, the polarization is longitudinal. The previous calculations, 6,7,9,10 giving transverse polarization, correspond to the point $W_{1}=W_{2}=0$. Thus the contribution of the channels with $m_{l}= \pm 1$ "tunes" the interaction to provide the experimentally observed longitudinal polarization in PuSb.

The change in polarization direction can be understood by detailed examination of the matrix elements giving the anisotropy of the hybridization-mediated two-ion interactions. Figures 2 and 3 illustrate this by showing some of these matrix elements (as defined in Ref. 6). Here $\theta$ is the angle between the moment direction and the interionic axis. As shown in Fig. 2, the original calculations for $L-S$ coupled $\mathrm{Pu}^{3+}$ (including only the $m_{l}=0 \leftrightarrow m_{l}=0$ 
channel) favored moment alignment parallel to the interionic axis, i.e., the maximum magnitude of the matrix elements occurs at $\theta=0$. This is opposite to the behavior for $\mathrm{Ce}^{3+}$, where moment alignment perpendicular to the interionic axis is favored. However, as shown in Fig. 3, as the $m_{l}=0 \leftrightarrow m_{l}= \pm 1$ and/or $m_{l}= \pm 1 \leftrightarrow m_{l}= \pm 1$ channels are opened up, the anisotropy for $\mathrm{Pu}^{3+}$ rapidly reverses direction to become ceriumlike, and this explains the change in polarization.

We have calculated the temperature dependence of the ordered moment for an fcc $\mathrm{Pu}^{3+}$ lattice with parameters characteristic of PuSb, as shown in Fig. 4 together with the experimental magnetization results of Burlet et al. ${ }^{12}$ We have included the crystal-field interaction, given for a cubic system as, ${ }^{28}$

$$
\mathcal{H}_{\mathrm{CF}}=\mathrm{B}_{4}\left(\mathrm{O}_{4}^{0}+5 \mathrm{O}_{4}^{4}\right) \text {. }
$$

Here $O_{m}^{n}$ are the Stevens operators, ${ }^{29}$ the crystal-field splitting $\Delta_{\mathrm{CF}}=360 B_{4}$, and a negative $B_{4}$ yields a $\Gamma_{8}$ ground state as is experimentally observed. ${ }^{11}$ For the antiferromagnetic phase $(+++---)$, we have plotted the average of the moments of the three ferromagnetically aligned planes. For intermediate coupling the value of $E_{1}\left(E_{1}=149 \mathrm{~K}\right)$ was chosen to match the experimental value of $T_{N}(85 \mathrm{~K})$ for $\mathrm{PuSb}$, and $E_{3} / E_{1}=-0.279$ was chosen to fit the transition temperature $(67 \mathrm{~K})$ from the ferromagnetic to the long-period antiferromagnetic struc-

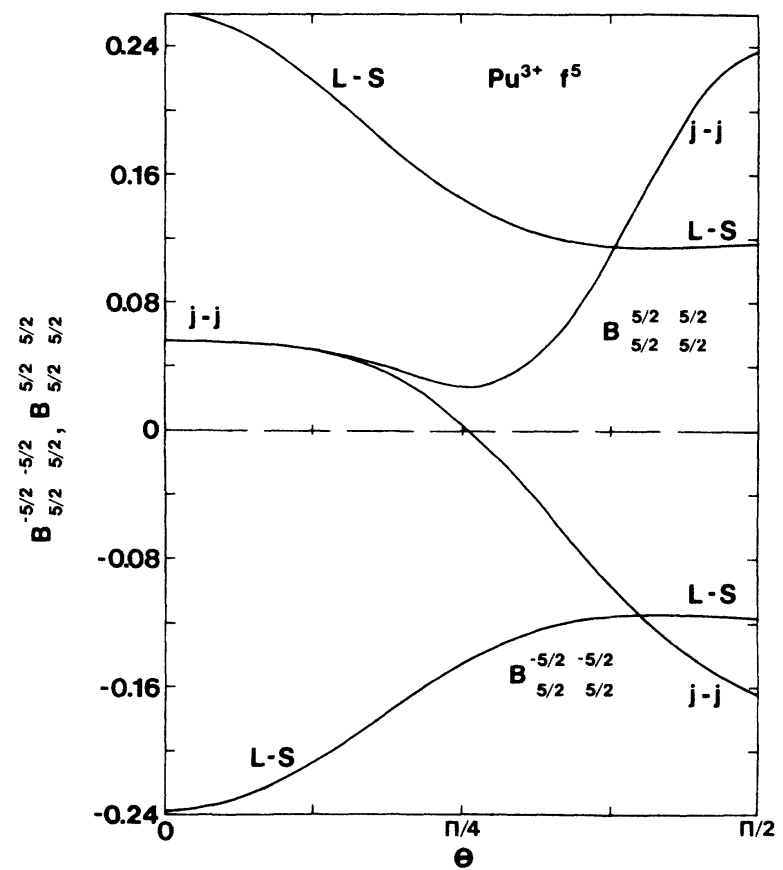

FIG. 2. The angular dependence of the two-ion diagonal matrix element $B_{\mu \nu}^{\epsilon \sigma}$ [defined in Eq. (9)] for coupling between $\mathrm{Pu}^{3+}$ ions with saturated parallel and antiparallel moments. For the $L-S$ case, the only effects included are those from the $m_{l}=0 \leftrightarrow m_{l}=0$ scattering channel. The upper indices label the $M_{J}$ states on one ion, and the lower indices label the states of the other ion. $\theta$ is the angle between the interionic axis and the axis of moment alignment. [The $j$-j curves are identical (Refs. 6 and 7) to the matrix elements for $\mathrm{Ce}^{3+} f^{1}$.] ture. Although the magnitude of moment depends slightly on the choice of the crystal-field parameter $B_{4}$, and a rather large crystal field $\left[\Delta_{\mathrm{CF}}=1200 \mathrm{~K}\right.$, or $60 B_{4}$ $\left.=-(1.3) E_{1}\right]$ will yield the experimentally correct moment for the IC calculation, we preferred to choose the value of $B_{4}$ to produce two polarized branches ${ }^{14}$ in the excitation spectrum at the zone boundary as we will discuss below in Sec. IV C. The thermal variation of the ordered moment was also obtained for the case of $L-S$ coupling, which is also shown in Fig. 4, for $E_{2}=E_{1}$, $E_{3}=-0.306 E_{1}, B_{4}=-(0.38 / 60) E_{1}$, and $E_{1}=121 \mathrm{~K}$ with $W_{1}=1, W_{2}=0$. Because of the difference of the $g$ factor ( $\frac{2}{7}$ for the $L-S$ coupling ground state and $\frac{5}{14}$ for the IC ground state with $75 \%{ }^{6} \mathrm{H}$ and $25 \%{ }^{4} \mathrm{G}$ ) the absolute values of moment for IC are larger than those for the $L-S$ coupling case. At zero temperature the theoretically pre-

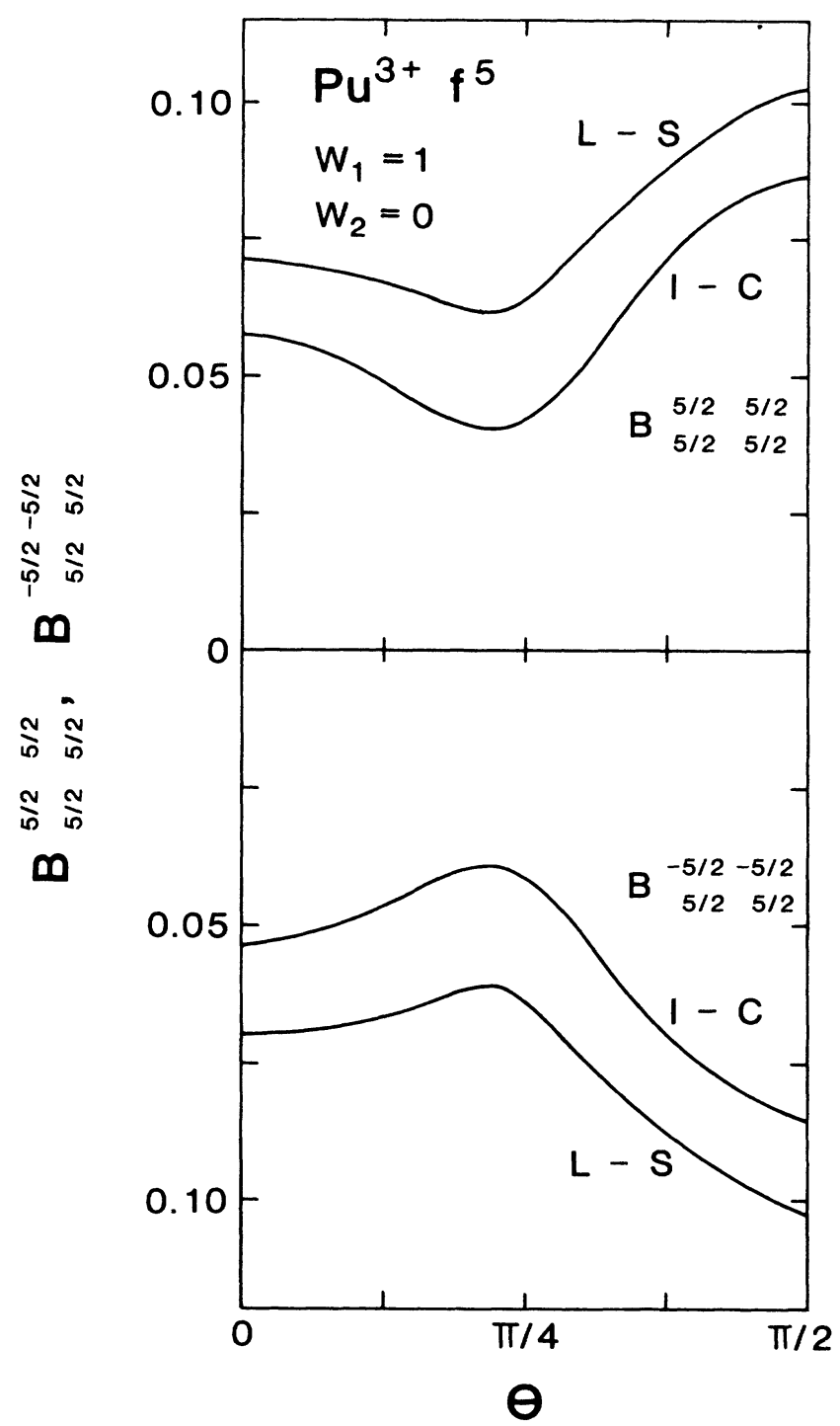

FIG. 3. The angular dependence of the same two matrix elements between $\mathrm{Pu}^{3+}$ ions as in Fig. 2, but now including a contribution from the resonant scattering channels involving $m_{l}= \pm 1$. The matrix elements are shown for both $L-S$ and IC within the $\mathrm{Pu}^{3+}$ ion. 
dicted moment is $0.677 \mu_{B}$ for the $L-S$ coupled state and $0.848 \mu_{B}$ for the IC state, while the experimental value of the $f$ moment is $(0.76 \pm 0.03) \mu_{B}$ determined from neutron scattering by Lander et al. ${ }^{11}$ Presumably the theory will predict an even larger moment for the exact IC case (rather than our approximation of including only ${ }^{6} \mathrm{H}$ and ${ }^{4} G$ components) because of the larger $g$ factor $(g=0.415)$.

\section{B. Correlation-length anisotropy}

The ratio $\kappa_{\|} / \kappa_{\perp}$ of the inverse critical correlation lengths parallel and perpendicular to the moment direction, evaluated at $T-T_{N}=0.04 T_{N}$, with negative crystal-field splitting ( $\Gamma_{8}$ low) as is experimentally observed $^{11}$ has been calculated using the methodology of Ref. 13. This is shown in Fig. 5 for the case of a longitudinally polarized antiferromagnetic structure for the IC case with $W_{1}=1, W_{2}=0, E_{2}=E_{1}, E_{3}=-0.279 E_{1}$. These parameters provide equilibrium behavior for $\mathrm{PuSb}$ closely approximating the experimental behavior. We find that $\kappa_{\|} / \kappa_{1}$ is almost independent of the crystal field, varying between 2.2 and 2.3 over a wide range of crystalfield values as shown in Fig. 5. [For $L-S$ coupled $\mathrm{Pu}^{3+}$ (with $W_{1}=1, W_{2}=0, E_{2}=E_{1}, E_{3}=-0.306 E_{1}$ ), the behavior is quite similar with $\kappa_{\|} / \kappa_{\perp}$ being about 2.0.] The value of $\kappa_{\|} / \kappa_{\perp}$ is greater than unity, indicating that the magnetic interactions within the ferromagnetic (100)

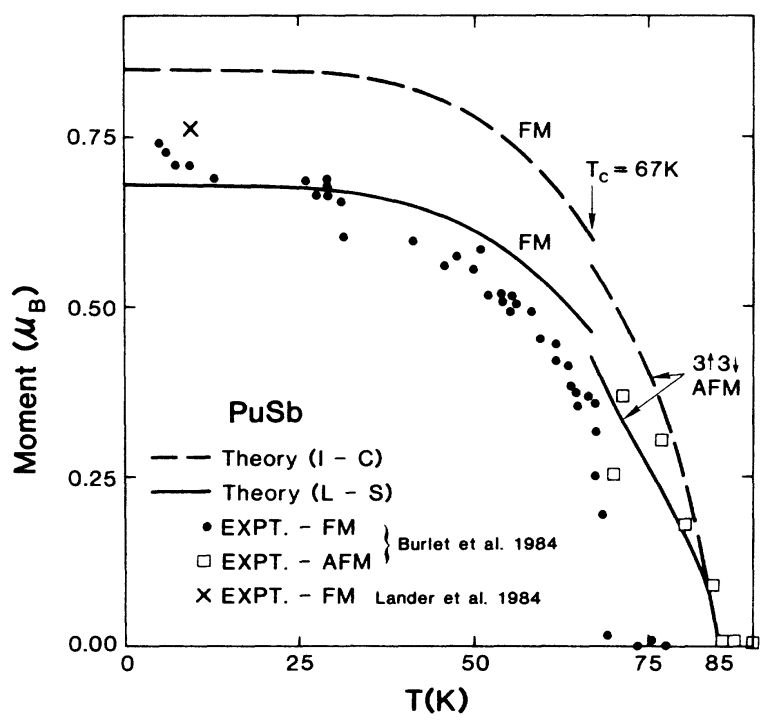

FIG. 4. Variation of ordered magnetic moment with temperature. The experimental magnetization values are from the neutron-diffraction measurements of Burlet et al. (Ref. 12). We also show the low-temperature experimental ordered $f$ moment from the neutron experiments of Lander et al. (Ref. 11). The theoretical curves show the predicted ordered $f$ moment for the intermediate-coupling case (with $E_{2}=E_{1}, E_{3}=-0.279 E_{1}$, and $60 B_{4}=-0.31 E_{1}$; to match the experimental value of $T_{N}=85 \mathrm{~K}$ for PuSb, $E_{1}=149.1 \mathrm{~K}$ ) and for the $L-S$ coupling case (with $E_{2}=E_{1}, E_{3}=0.306 E_{1}, 60 B_{4}=-0.38 E_{1}$, and $E_{1}=121.4 \mathrm{~K}$ ). The average sublattice moment has been plotted for the antiferromagnetic phase. planes are stronger than are the interplanar couplings. This result is in close agreement with that of recent experiments $^{27}$ giving $\kappa_{\|} / \kappa_{1}=1.8 \pm 0.2$ for $\mathrm{PuSb}$. We note two important changes from the previous results ${ }^{13}$ that did not include any contribution from scattering channels (2) and (3) (the $m_{l}= \pm 1$ channels). The singularity in the near-critical susceptibility now provides the correct polarization as experimentally observed; and the direction of anisotropy for both IC and $L-S$ coupling is reversed from that previously found $\left(\kappa_{\|} / \kappa_{1}>1\right.$ as has now been experimentally observed ${ }^{27}$ ).

\section{Magnetic excitation behavior}

Using the same random phase approximation (RPA) equations of motion technique as previously, ${ }^{9,10}$ we have calculated the magnetic excitation behavior predicted for $\mathrm{PuSb}$. This involves taking the difference between the exact Hamiltonian [given by the sum of the hybridizationmediated exchange Hamiltonian of Eq. (7) and the crystal-field Hamiltonian of Eq. (19)] and the mean-field approximation to that Hamiltonian. This difference is then treated as a perturbation of the mean-field Hamiltonian to obtain the equations of motion given by Eq. (12). The resulting predicted excitation spectra and intensities of the modes for PuSb are shown in Figs. 6 and 7 for the $L-S$ and IC cases, respectively. (Since the "bare" crystal field is strongly modified ${ }^{30}$ by the hybridization pushing the $\Gamma_{8}$ level below the $\Gamma_{7}$ level in energy, for the excitation calculations as well as for the equilibrium and

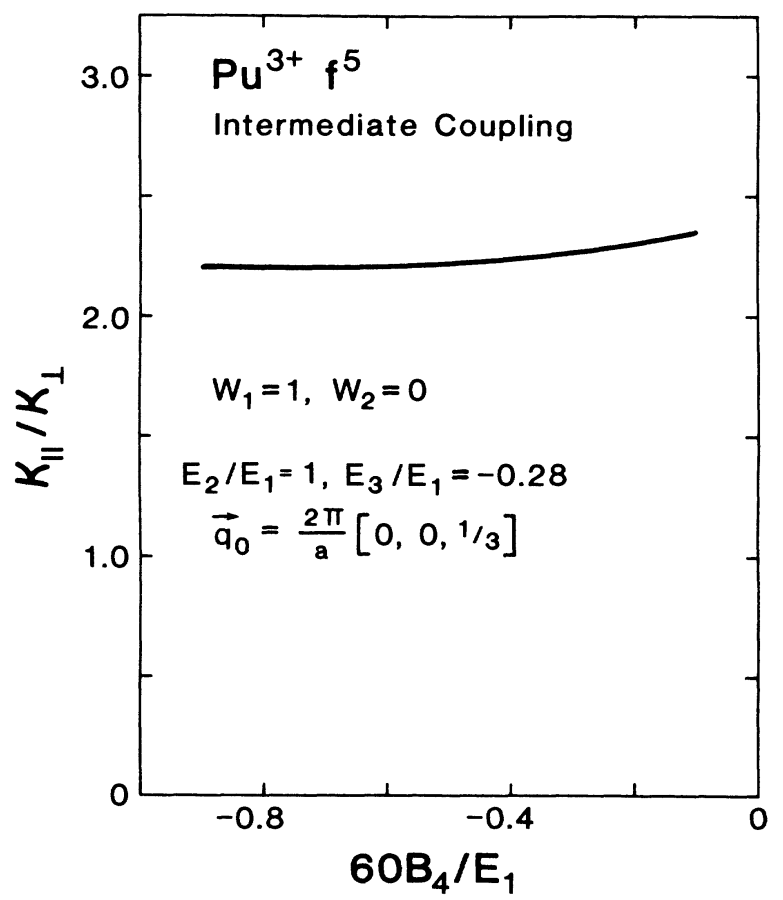

FIG. 5. Ratio of inverse correlation lengths parallel and perpendicular to the moment direction for parameters characteristic of PuSb, plotted as a function of the ratio of the crystal-field parameter $60 \mathrm{~B}_{4}$ to the nearest-neighbor anisotropic exchange parameter $E_{1}$. 

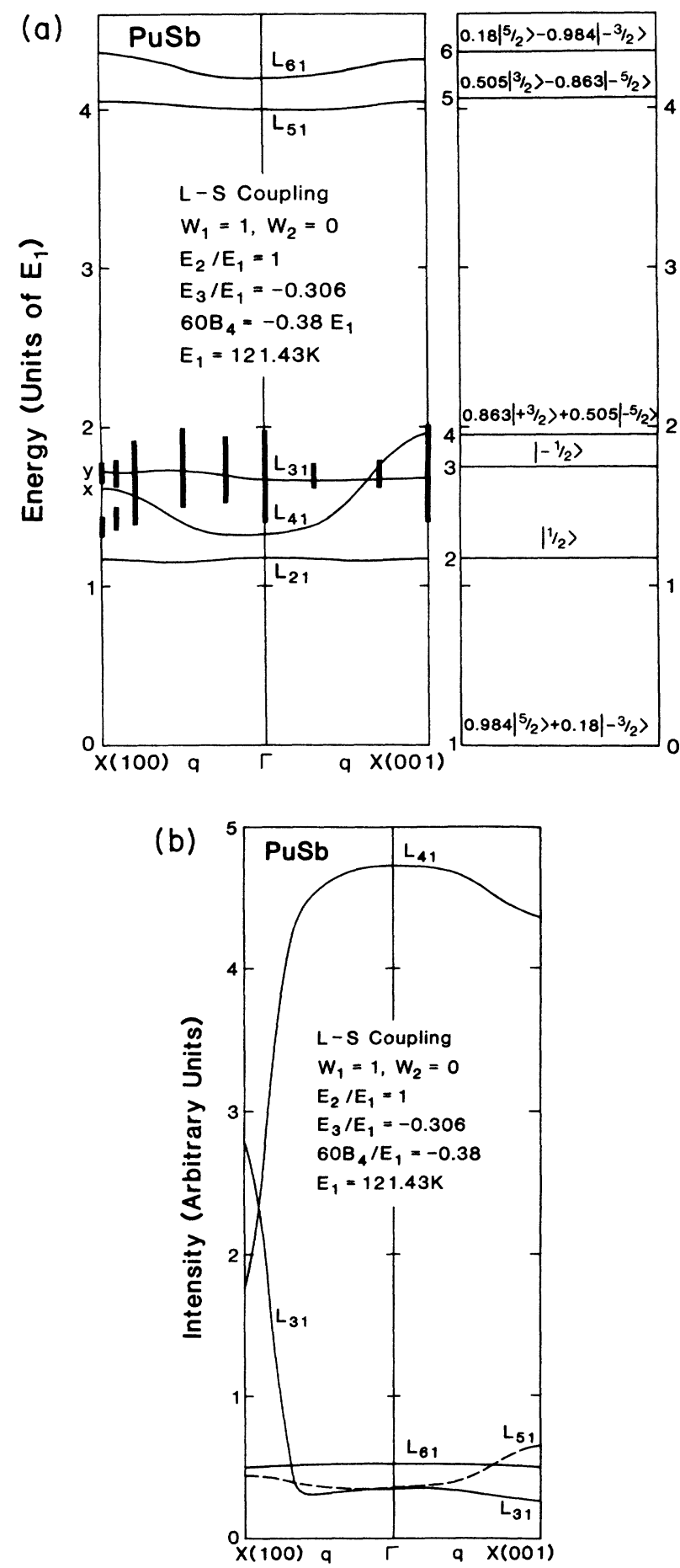

FIG. 6. (a) Dispersion curves and (b) intensities for excitations in the ferromagnetic phase of $\mathrm{PuSb}$ at $T=0$ with $L-S$ coupling, $E_{2}=E_{1}, \quad E_{3}=0.306 E_{1}$, and $60 B_{4}=-0.38 E_{1}$ with $W_{1}=1, W_{2}=0$ for $\mathbf{q}$ along the [100] and [001] (directions parallel and perpendicular to the moment direction, respectively). $E_{1}=121 \mathrm{~K}$ to match experimental $T_{N}$. Modes $L_{31}, L_{41}$, and $L_{51}$ are transverse modes (i.e., time-dependent mode moment transverse to the equilibrium moment) and modes $L_{61}$ and $L_{21}$ are longitudinal and quadrupolar modes, respectively. The energy levels of the molecular-field states and their compositions in terms of the angular momentum eigenstates (quantized along [001]) are shown on the far right. Heavy bars show the experimental results of Lander et al. (Ref. 14) at $10 \mathrm{~K}$ for PuSb. correlation length calculations above, we have treated the crystal-field parameter $B_{4}$ phenomenologically and omitted its temperature dependence. ${ }^{31}$ )

The dominant mode in intensity across most of the Brillouin zone is the $L_{41}$ mode which has an excitation energy of about $3.4 \mathrm{THz}(164 \mathrm{~K})$ at the $\Gamma$ point for both $L-S$ coupling and IC. The $L_{41}$ mode energy increases on

(a)

Intermediate Coupling

$W_{1}=1, W_{2}=0, E_{2} / E_{1}=1, E_{3} / E_{1}=-0.279$

$60 B_{4} / E_{1}=-0.31, E_{1}=149.12 K$

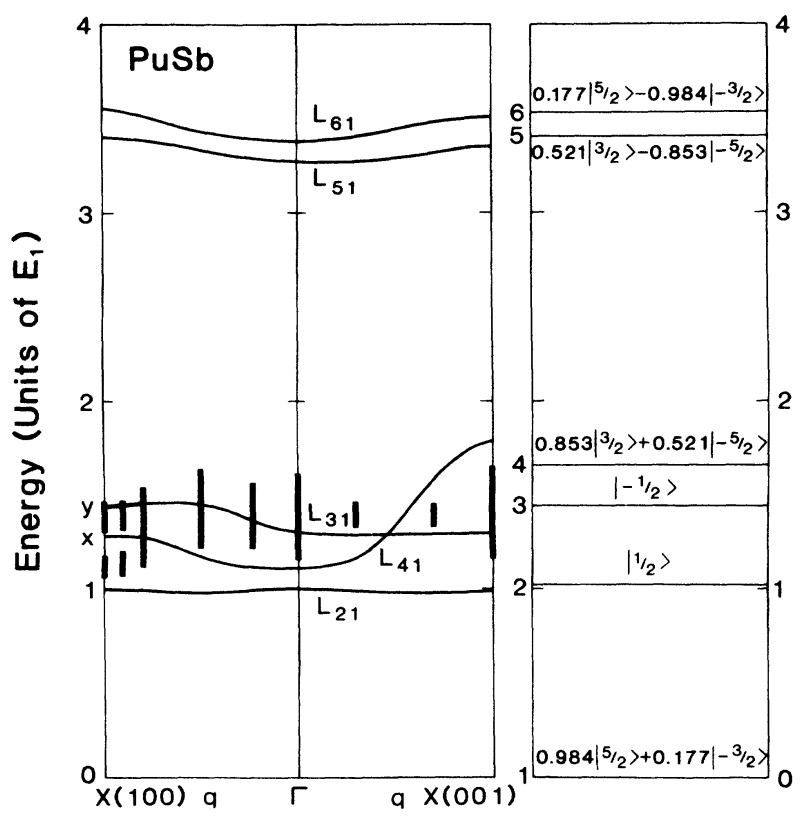

(b)

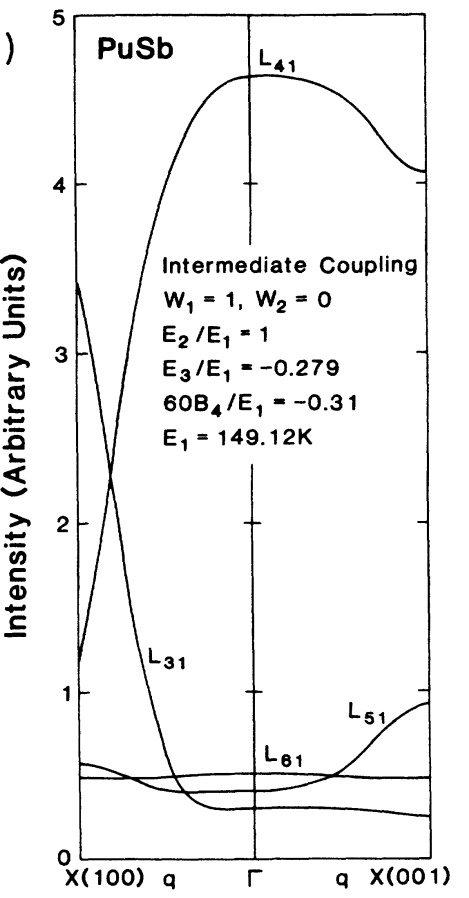

FIG. 7. (a) Dispersion curves and (b) intensities of PuSb as in Fig. 6, but now calculated for intermediate coupling within the $\mathrm{Pu}^{3+} f^{5}$ ion. 
going toward the $X$ points. At about $(0.7,0,0)$, mixing of the transverse modes $L_{31}$ and $L_{41}$ starts to occur. There is an associated reduction of the intensity of the $L_{41}$ mode, and a corresponding increase in the $L_{31}$ mode intensity. This admixture produces two elliptically polarized branches (with mode moment transverse to equilibrium moment): a $y$-polarized (major axis of timedependent mode moment perpendicular to q) upper branch and an $x$-polarized (major axis of time-dependent mode moment parallel to $q$ ) lower branch. At the zone boundary ( $q \|[100]$ direction), the two predicted modes in the case of $L-S$ coupling have energies $4.5 \mathrm{THz}(216 \mathrm{~K})$ and $4 \mathrm{THz}(194 \mathrm{~K})$ respectively, and the polarization $\left(\left\langle J_{y}^{2}\right\rangle /\left\langle J_{x}^{2}\right\rangle\right)^{1 / 2}$ is $\sqrt{2}$ for the upper branch and $(1 / \sqrt{6})$ for the lower branch. In the IC case, the energies of the two modes are 4.4 THz $(218 \mathrm{~K})$ and 4.0 THz $(191 \mathrm{~K})$, and the polarization is $\sqrt{6}$ for the upper branch and $1 / \sqrt{2}$ for lower branch.

The relative separations between modes are quite sensitively dependent on the value of $B_{4}$. To bring modes $L_{31}$ and $L_{41}$ close enough to cause the admixture at the zone boundary (i.e., to give two intense modes) the value of $60 B_{4}$ must be chosen in a narrow range from $-0.35 E_{1}$ to $-0.40 E_{1}$ for the $L-S$ coupling and from $-0.25 E_{1}$ to $-0.31 E_{1}$ for IC. Outside this range of $60 B_{4}$ the only intense mode is $L_{41}$, and it is circularly polarized. In fact, we chose $B_{4}$ to provide the mode mixing giving two intense (and elliptically polarized) modes at the zone boundary. It is a remarkable result that this choice of crystal-field splitting parameter places the mode energies almost exactly at the experimental values. This result indicates that for PuSb the crystal-field splitting from $\Gamma_{8}$ to $\Gamma_{7}$ should be about $255-290 \mathrm{~K}$. Further experimental information checking this predicted value would be valuable.

Note that we found almost the same results for the $L-S$ coupling and intermediate coupling IC cases. This is not surprising since the ground state of the $L-S$ coupling limit is the predominant component of the IC ground state which we take as $75 \%{ }^{6} \mathrm{H}$ and $25 \%{ }^{4} \mathrm{G}$.

One major discrepancy exists between the calculated and observed excitation behavior at low temperature. While the experimentally observed magnetic excitation spectrum is almost dispersionless for $q$ across most of the Brillouin zone, and a clearly defined minimum occurs for the low-energy branch at the zone boundary ( $X$ point), our theory predicts that the intense mode has significant dispersion for $q$ along both the [001] and [100] directions with an energy minimum at the $\Gamma$ point regardless of the choice of phenomenological parameters. It is interesting that the $L_{31}$ mode, which shares the intensity at the $X$ point is essentially dispersionless with a shallow minimum at the [100] $X$ point. Thus it may be that this remaining discrepancy lies in the rather sensitive nature of the intensity sharing between the $L_{31}$ and $L_{41}$ modes.

We have also extended our excitation calculations to finite temperatures using the random phase approximation. The mode intensity distribution at the [100] zone boundary so obtained is shown in Fig. 8. (These calculations include a higher-order decoupling of the equations

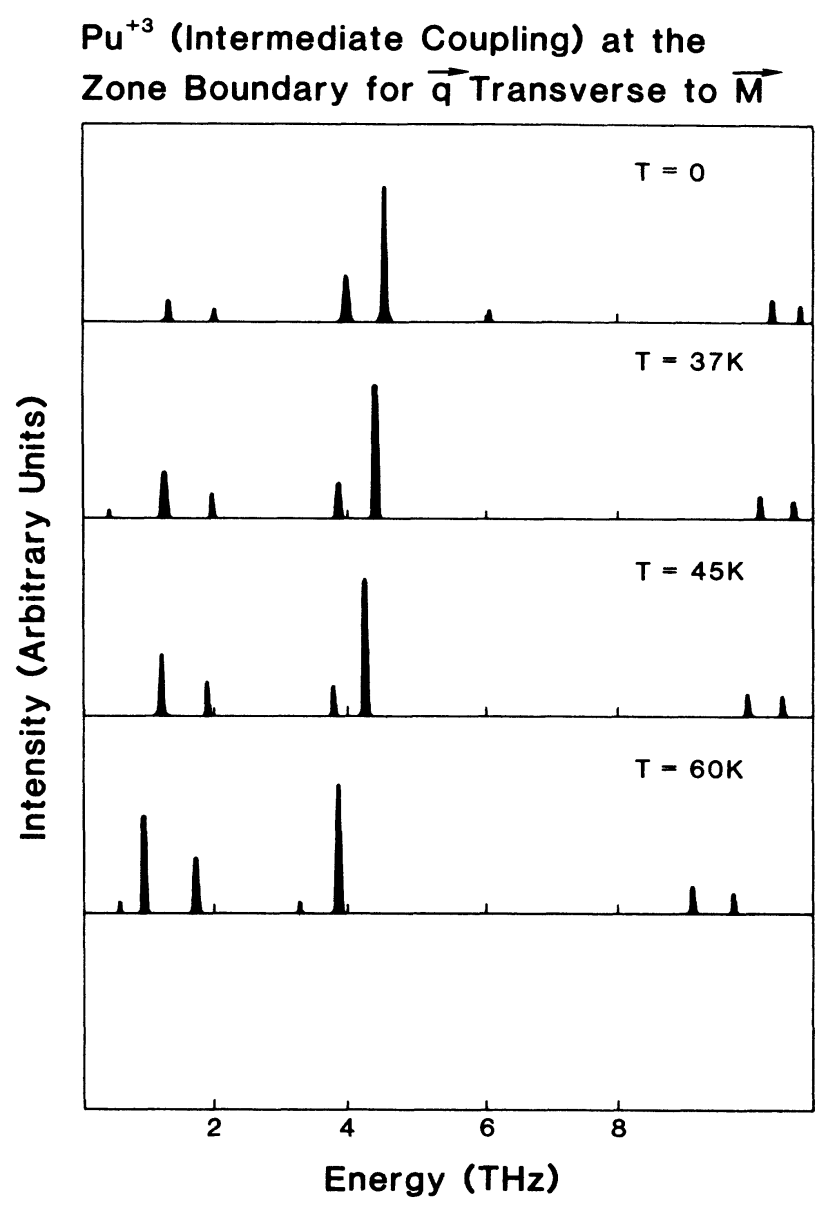

FIG. 8. Predicted temperature dependence of the excitation mode intensities at the [100] Brillouin-zone boundary for PuSb.

of motion than that used to obtain Figs. 6 and 7, so at $T=0$ some additional low intensity modes are found.) The main effect predicted is a filling in of intensity at the low energy end of the spectrum as temperature increases.

\section{Summary}

In conclusion, we have found that upon including the additional (next-to-dominant) channels for the single-site scattering event the hybridization-mediated anisotropic two-ion interaction fully explains the experimental anisotropic magnetic equilibrium behavior ${ }^{8,11,12}$ for the fcc $\mathrm{Pu}^{3+} f^{5}$ system pertinent to $\mathrm{PuSb}$. The longitudinally polarized antiferromagnetic phase has been satisfactorily obtained by considering the contribution of the additional scattering channels, which is equivalent to including some additional off-interionic-axis $f$-electron charge distribution. We also obtain excellent agreement with experiment $^{27}$ for the correlation length anisotropy. The theory also gives a magnetic excitation spectrum at $T=0$ in the ferromagnetic phase in close agreement with the observed 
energies, ${ }^{14}$ with two polarized branches at the zone boundary with $\mathbf{q}$ along the [100] (transverse-to-moment) direction as is experimentally observed. ${ }^{14}$ The predicted crystal-field splitting from $\Gamma_{8}$ to $\Gamma_{7}$ is about 255 to $290 \mathrm{~K}$. The remaining discrepancy with experiment is that we predict that the major mode intensity across the Brillouin zone is not in a largely dispersionless mode, and our lower-energy mode at the zone boundary does not occur at an energy minimum.

\section{ACKNOWLEDGMENT}

This research has been supported by the U.S. Department of Energy through Grant No. DE-FG05-84ER45134. G. H. Lander has provided exceptionally valuable aid through his continuing interest as well as providing information on the experimental situation. We have benefited from information and discussion from P. Burlet, J. Rossat-Mignod, and O. Vogt.
"Present address: Department of Physics, California State University at Northridge, Northridge, CA 91330.

†Present address: NASA Lewis Research Center, Cleveland, OH 44135.

${ }^{1}$ B. R. Cooper and R. Siemann, J. Appl. Phys. 50, 1991 (1979); R. Siemann and B. R. Cooper, Phys. Rev. Lett. 44, 1015 (1980); B. R. Cooper, J. Magn. Magn. Mater. 29, 230 (1982).

${ }^{2}$ D. Yang and B. R. Cooper, J. Appl. Phys. 53, 1988 (1982); B. R. Cooper, P. Thayamballi, and D. Yang, ibid. 55, 1866 (1984); P. Thayamballi, D. Yang, and B. R. Cooper, Phys. Rev. B 29, 4049 (1984).

${ }^{3}$ N. Kioussis, B. R. Cooper, and A. Banerjea, J. Appl. Phys. 61, 3388 (1987); Phys. Rev. B (to be published).

${ }^{4}$ J. M. Wills and B. R. Cooper, Phys. Rev. B 36, 3809 (1987).

${ }^{5}$ B. Coqblin and J. R. Schrieffer, Phys. Rev. 185, 847 (1969).

${ }^{6}$ B. R. Cooper, R. Siemann, D. Yang, P. Thayamballi, and A. Banerjea, in Handbook on the Physics and Chemistry of the Actinides, edited by A. J. Freeman and G. H. Lander (NorthHolland, Amsterdam, 1985), Vol. 2, Chap. 6, pp. 435-500.

${ }^{7}$ P. Thayamballi and B. R. Cooper, J. Appl. Phys. 55, 1829 (1984).

${ }^{8}$ B. R. Cooper, P. Thayamballi, J. C. Spirlet, W. Muller, and O. Vogt, Phys. Rev. Lett. 51, 2418 (1983).

${ }^{9}$ A. Banerjea, B. R. Cooper, and P. Thayamballi, Phys. Rev. 30, 2671 (1984).

${ }^{10}$ A. Banerjea and B. R. Cooper, Phys. Rev. B 34, 1607 (1986).

${ }^{11}$ G. H. Lander, A. Delapalme, P. J. Brown, J. C. Spirlet, J. Rebizant, and O. Vogt, Phys. Rev. Lett. 53, 2262 (1984); J. Appl. Phys. 57, 3748 (1985).

12P. Burlet, S. Quezel, J. Rossat-Mignod, J. C. Spirlet, J. Rebizant, W. Muller, and O. Vogt, Phys. Rev. B 30, 6660 (1984).

${ }^{13}$ N. Kioussis and B. R. Cooper, Phys. Rev. B 34, 3261 (1986).

${ }^{14}$ G. H. Lander, W. G. Stirling, J. Rossat-Mignod, J. C. Spirlet, J. Rebizant, and O. Vogt, Physica 136B, 409 (1986).

${ }^{15}$ See discussion on p. 1612 in the Appendix of Ref. 10. While the $j=\frac{7}{2}$ single-electron states contribute to both the $L-S$ and IC $J=\frac{5}{2}$ ground-state multiplet of $\mathrm{Pu}^{3+}$, recent first- principles calculations (Ref. 4) indicate that they contribute one percent or less to the pertinent range parameters (exchange constants). Thus the neglect of the $j=\frac{7}{2}$ contribution to the scattering amplitudes seems well justified, and we do this. In any case, in the present treatment, the relative contributions of the different scattering channels to the two-ion interaction are treated as phenomenological parameters.

16P. W. Anderson, Phys. Rev. 124, 41 (1961).

${ }^{17}$ B. Cornut and B. Coqblin, Phys. Rev. B 5, 4541 (1972).

18J. R. Schrieffer, J. Appl. Phys. 38, 1143 (1967).

19J. R. Schrieffer and P. A. Wolff, Phys. Rev. 149, 491 (1966).

${ }^{20}$ M. A. Ruderman and C. Kittel, Phys. Rev. 96, 99 (1954); T. Kasuya, Prog. Theor. Phys. 16, 45 (1956); K. Yosida, Phys. Rev. 106, 893 (1957).

${ }^{21}$ B. R. Cooper, G.-J. Hu, N. Kioussis, and J. M. Wills, J. Magn. Magn. Mater. 63 - 64, 121 (1987).

${ }^{22}$ B. R. Cooper, J. Less-Common Met. 133, 31 (1987).

${ }^{23} \mathrm{M}$. Tinkham, Group Theory and Quantum Mechanics (McGraw-Hill, New York, 1964), Chap. 5.

${ }^{24}$ B. Judd, Operator Techniques in Atomic Spectroscopy (McGraw-Hill, New York, 1963).

${ }^{25}$ W. T. Cornall and B. G. Wybourne, J. Chem. Phys. 40, 3428 (1964).

${ }^{26}$ G. Racah, Phys. Rev. 61, 186 (1942); 62, 438 (1942); 63, 367 (1943); 76, 1352 (1949).

${ }^{27}$ P. Burlet, J. Rossat-Mignod, G. H. Lander, J. C. Spirlet, J. Rebizant, and O. Vogt, Phys. Rev. B 36, 5306 (1987).

${ }^{28}$ M. T. Hutchings, in Solid State Physics, edited by F. Seitz and D. Turnbull (Academic, New York, 1964), Vol. 16, pp. 227-273.

${ }^{29}$ K. W. H. Stevens, Proc. Phys. Soc. London, Sect. A 65, 209 (1956).

30J. M. Wills and B. R. Cooper, J. Magn. Magn. Mater. 54 - 57, 1049 (1986).

${ }^{31}$ P. Thayamballi and B. R. Cooper, Phys. Rev. B 30, 2931 (1984). 